\title{
New Manifestation of Covid-19 in Oral Region, a Potential Faster Diagnosis Approach, a Mini Review
}

\author{
Babak Baban', Mark R Stevens ${ }^{2}$, Shohreh Ghasemi ${ }^{3,}$, Fargol Mashhadi Akbar Boojar ${ }^{4}$ and Mahmood Dashti $^{5}$ \\ ${ }^{1}$ Professor and Associate Dean for research the Dental College of Georgia, USA \\ ${ }^{2}$ Chief of OMFS Department of Augusta University, GA, USA \\ ${ }^{3}$ Adjunct assistant professor in Department of oral and maxillofacial surgery in University of Augusta, GA, USA \\ ${ }^{4}$ Dentistry student, research committee of Golestan Medical University, Iran \\ ${ }^{5}$ Private practice of Dental Med Spa, Tehran, Iran
}

*Corresponding author: Shohreh Ghasemi, Adjunct assistant professor in Department of oral and maxillofacial surgery in University of Augusta, GA, USA

Received date: 11 June, 2021 | Accepted date: 9 July, 2021 | Published date: 13 July, 2021

Citation: Baban B, Stevens MR, Ghasemi S, Boojar FMA, Dashti M (2021) New manifestation of Covid-19 in oral region, a potential faster diagnosis approach, a mini review. J Dent Oral Epidemiol 1(1). doi https://doi.org/10.54289/JDOE2100103

Copyright: () 2021 Baban B, et al. This is an open-access article distributed under the terms of the Creative Commons Attribution License, which permits unrestricted use, distribution, and reproduction in any medium, provided the original author and source are credited.

\begin{abstract}
Introduction: The pandemic of COVID-19 continues to shatter the world by high rate of mortalities. The poorest prognosis of COVID-19 is among patients with comorbidities. Exploring new ways to predict and diagnose the infection at early stages will probably reduce the fatality and prevent further damages. Identifying unexampled or additional clinical symptoms may provide opportunities for earlier diagnosis and prevention of severe cases of COVID-19.

Material and method: A methodical search was conducted through PubMed, Google and Google scholar databases to analyze the published or in press literature. The keywords were words "novel Coronavirus", "SARS-Cov-2 (CoV)" and "COVID-19", searched by at least two or more independent individuals. A total of 88 related items were picked based on the keywords. While the majority of materials were based on one-case reports (66 cases), a combination of multiple case studies as well as full research papers was investigated.

Results: There were several unexampled manifestations including cutaneous symptoms and oral lesions by which early diagnosis, prevention and even more effective treatment may be achieved for COVID-19 patients.

Conclusion: The most striking novel clinical presentation was intraoral painful blisters or mouth ulcers mostly seen on the cheek mucosa as per the revelation of the reported cases in the literature review. Leaving out a daily intraoral examination might have been the reason why intraoral lesions have been connived as effective predictive, prognostic and even preventive manifestations of COVID-19. The outcomes of these current studies suggest that intraoral lesions may offer a very new approach, using preventive, predictive and personalized medicine (PPP) concept to provide a rapid diagnosis and more effective therapeutic modality in patients with COVID-19.
\end{abstract}

Keywords: COVID-19; Oral lesions; SARS-CoV-2; PPP; Preventive; coronavirus; Personalized

Abbreviations: PPP: preventive predictive and personalized medicine, SARS: Severe Acute Respiratory Syndrome, COVID: corona virus disease. 


\section{Introduction}

The well-being of the world has been affected to a large extent due to the occurrence and fast outbreak of the coronavirus and the pandemic of COVID-19. The complex nature of SARSCov-2, the virus causing COVID-19 and its high transmissibility has been unprecedentedly unique. Currently, other than supportive therapy, there is no definitive treatment for patients with COVID-19. That is why earlier diagnosis would provide not only better prognosis for COVID-19 patients, but also would prevent the transmission of the infection to healthy individuals significantly. Therefore, adopting a preventive, predictive and personalized (PPPs) medicine approach maybe a highly effective way to mitigate the burden of COVID-19 at an individual level as well as for healthcare system globally. By providing reliable criteria and standards, it would be potentially possible to predict and diagnose the infection at earlier stages in a personalized fashion. Abating the rate of mortality and hospitalization as well as preventing new infections would be the most divulged consequences of a PPPs strategy in fight against COVID-19. Since January $31^{\text {st }} 2020$ when COVID-19 was officially announced as a global health emergency and later on a worldwide pandemic by WHO on March $11^{\text {th }}, 2020$, the guidelines on symptoms and diagnostic indices have been continuously updated. Currently, fever, cough, tiredness and shortness of breath, headache, chest pain, loss of taste/smell, muscle aches and sore throat are among most common clinical manifestations reported in symptomatic COVID-19 patients. Since timing is highly critical considering the fast track of transmission of SARS-Cov-2, it is utterly plausible to explore unique and maybe specific manifestations to accelerate the diagnosis and improve the preventive and therapeutic measures. The goal of this report is to introduce intraoral lesions as unexampled clinical presentations during COVID-19 infection. These oral symptoms maybe good indicators to help clinicians and scientists to diagnose early and may be effective in preventing new cases. Most important oral lesions are relatively easier to be detected and monitored by patients at individual level, as an excellent example of predictive, preventive and personalized medicine (PPPs).

\section{Method and Material:}

The study was to conduct an extensive search through databases comprising CovLit, PubMed, and the National Institute of Health for SARS-CoV-2. Keywords including SARS-CoV-2 and its cutaneous symptoms in the intraoral cavity with pleomorphic clinical manifestations. The inclusion criteria were to consider only those topics describing symptoms merely caused by SARS-CoV-2 virus published by August 2020. Reports and materials addressing all other iatrogenic factors and side effects related to the treatment were excluded.

\section{Results:}

A sum of 181 articles was initially selected to be screened. After thoroughly analysis only 11 articles met the standards, based on the inclusion/exclusion criteria, to be included in the final stage of the study.

\section{Discussion:}

The majority of studies and articles examined for this current review reported a set of common symptoms for the most cases of COVID-19. Diarrhea, dyspnea, pneumonia, sore throat, hypogeusia, hyposmia, and headaches were the most usual signs often mentioned. However, there were a certain level of disparities among responses from an individual to another based on genetic and epigenetic factors. These were including, not limited to, immune responses as well as proteomic and metabolic indices [1]. Further, being a pandemic with high transmissibility, COVID-19 presents an evident diverse spectrum of clinical manifestations based on the Geo-demographic Variabilities.

Cutaneous symptoms are a set of clinical manifestations were reported by several studies as dermatologic symptoms at the early stages and onset of COVID-19 indexed in Pubmed database [1,2]. Symptoms such as Livedoid eruptions, oral lesions, vesicular eruptions, morbilliform rash, urticaria and acral lesions are all examples of cutaneous manifestations. It is hinted that cutaneous symptoms maybe the early signs of COVID-19 infection [3-5]. In fact, this suggest that cutaneous lesions maybe used as a tool for early diagnosis by individuals, exemplifying a model of the application of predictive, preventive and personalized medicine (PPPs). Oral cavity consists of a small vestibule and a larger oral 
cavity proper. A wide number of dermatological conditions can affect the oral cavity. Study by Carreras-Presas et.al., introduces a case with vesiculobullous oral lesions in a patient with SARS-CoV-2 infection [6]. It is a 56-year-old male who was probably the first COVID-19 patients reported with intraoral lesions. Further, in addition to cardinal symptoms, it is indicated that some COVID-19 patients had sore throat and tenderness of the palate. Abrasions detected on testing were resembling recurring herpetic stomatitis. Topical antiseptics and Valaciclovir were authorized resulting in the elimination of lesions after 10 days [6-7]. The recurring ulcers were studied within a short time after the first signs of erythematous macular lesions. The probability of vasculitis seems to be the right description for these findings.

In another study, Tang et al [8] documented the Varicella-like skin lesions in COVID-19 patients. This could be linked to what Carreras-Presas et.al already reported [6] considering the commonality between the virulence of herpes zoster (herpes varicella-zoster- HVZ3) [9] and SARS-CoV-2.

Multiple inflammatory and vascular reactions such as erythematous rashes are among new additions to the symptoms presented in patients with COVID-19, demonstrating the inflammatory nature of COVID-19

$[3,4,5,10,11,12]$. Early stage symptoms and measuring inflammatory biomarkers maybe very effective way to predict and protect against COVID-19 effectively, translating the modality of diagnosis and prevention to a personalized form of medicine (PPPs).

ACE2 is being documented as the most important host cell receptor of 2019-nCoV that is mainly responsible for the prone cells being invaded by the virus. Multiple RNA-seq profiles from the twin public databases comprising the Functional Annotation of The Mammalian Genome Cap Analysis of Gene Expression (FANTOM5 CAGE) and The Cancer Genome Atlas (TCGA) was collected to study the possible transmission method of the 2019-nCoV virus on the mucosa of the oral cavity. ACE2 has been shown to be expressed on intraoral lesions and on the mucosa of the oral cavity [13]. Additionally, the single-cell transcriptomes were utilized to find and establish the truth of ACE2- expressing cell structure and the portion in the oral activity. The findings indicated that the ACE2 was enforced on the mucosa of the oral cavity. ACE2 was mostly implanted in epithelial cells of the tongue which generated significant interest in the field. Those discoveries have clarified the fundamental technique that the oral cavity is a potential vulnerable site for SARSCoV-2 infection cases especially at the initial stages. Dental medicine as one of the major front lines in the war against COVID-19 can effectively adopt these measures in a PPPs fashion as Predictive, Preventive and Personalized medicine with a high level of reliability [13-14]. In all the cases, skin abrasions appear as erythematous papules in the upper trunk, which eventually changes to erythematous-violaceous spots with a murky center and a pseudo-vesicle in the middle. The lesions noticeably synthesize in the back-end and then move to the limbs and faces within a week. The palms and soles seem to be spared [15]. The intraoral cavity in such individuals revealed petechiae and palatal macules with identical outcomes in all patients. Further findings such as normal basket-weave stratum corneum, with minor to moderate spongiosis in the epidermis were deduced in earlier studies [16]. The dermis displayed lymphocytic perivascular and interstitial infiltrate, dilated vessels filled with neutrophils, and extravasation of red blood cells. Cases with similar signs were treated with systemic corticosteroids. The ongoing undertaking of skin lesions happened within 2-3 weeks.

Dermatology would be the other potential high risk front regarding cutaneous lesions during COVID-19 pandemic $[8,17]$. While cutaneous manifestations could be used as predictive measure, however, some maybe challenging and require to be differentially diagnosed. As an example, Erythema Multiforme (EM) is documented in $90 \%$ of the patients with COVID-19 while in less than $10 \%$ of individuals are caused by drug-related factors. Other viruses such as Parvovirus B19, Coxsackie and Adenovirus may cause EM-like or target-like exanthem which can be differentiated from those related to SARS-CoV-2 virus through its structure and morphology $[18,19]$.

Regardless of their source and the causative factor, It is noteworthy to mention that oral lesions during COVID-19 infection reinforces the notion that including dentists in the transdisciplinary team to support the effort during COVID-19 pandemic is a crucial and necessary. It would be highly 
critical to review patients oral and dental hygiene in hospital and once they're discharged. Regarding COVID-19 cases, certain oral signs and indications must be acknowledged like thrush-like ulcers, dysgeusia, geographic tongue, petechiae, HSV-1 infection, traumatic ulcers, candidiasis and many others. Hence, there should be a prominence given to the critical dental clinical test of patients with contagious diseases in the ICU, and their need for help, managing their pain and their general well-being [20]. The concurrent enanthem and pseudo- vesicles is more indicative of a contagious cause rather than simply drug side effects, more research and groundwork are needed.

\section{Conclusion:}

Altogether, our study examining cases and literature review demonstrated that intraoral lesions such as blisters and painful oral ulcers mostly visible on the cheek mucosa could be early indicative of COVID-19 infection. Adopting a PPP strategy (Predictive, Preventive and Personalized Medicine) may help early diagnosis, prevention and more effective treatment with increased rate of survilanve and life quality for patients with COVID-19. The absence of daily intraoral tests resulted in the poor detection of intraoral lesions as documented here in this mini review. Conducting a simple oral examination even at a personal and individual level could prevent most extremities with indecisive pathological measures and diagnosis during COVID-19 pandemic. Therefore, embracing a PPP strategy by all individuals including healthcare system professionals (e.g., Physicians, dentists, nurses, para-medics, etc.) would be highly recommended to help early diagnosis, prevention and more effective treatment for patients with COVID-19 as well as all people during this catastrophic pandemic. Measures such as a routine intraoral examination could be a great example of PPP strategy with significant impact on the outcome of current bout against COVID-19 pandemics.

Conflict of Interest: None to mention.

\section{References:}

1. Nicholson KG, Kent J, Hammersley V, Cancio E (1997) Acute viral infections of upper respiratory tract in elderly people living in the community: comparative, prospective, population based study of disease burden. BMJ. 315(7115): 1060-1064.
2. Peeri NC, Shrestha N, Rahman MS, Zaki R, Tan Z, et al. (2020) The SARS, MERS and novel coronavirus (COVID-19) epidemics, the newest and biggest global health threats: what lessons have we learned? Int J Epidemiol 49(3): 717-726.

3. Chaux-Bodard A-G, Deneuve S, Desoutter A (2020) Oral manifestation of Covid-19 as an inaugural symptom? J Oral Med Oral Surg 26(2): 18.

4. Madjid M, Safavi-Naeini P, Solomon SD, Vardeny O (2020) Potential Effects of Coronaviruses on the Cardiovascular System: A Review. JAMA Cardiol 5(7): 831-840.

5. de Carvalho LF das CES, Kitakawa D, Cabral LAG (2021) Oral lesions of herpes zoster in COVID-19 patients or truly associated to the disease? Oral Dis 27(3): 774-775.

6. Carreras-Presas CM, Sánchez JA, López-Sánchez AF, Jané-Salas E, Pérez MLS (2021) Oral vesiculobullous lesions associated with SARS-CoV2 infection. Oral Dis. 27(3): 710-712.

7. Biadsee A, Biadsee A, Kassem F, Dagan O, Masarwa S, et al. (2020) Olfactory and Oral Manifestations of COVID-19: Sex-Related Symptoms-A Potential Pathway to Early Diagnosis. Otolaryngol Head Neck Surg.163(4): 722-728.

8. Tang K, Wang Y, Zhang H, Zheng Q, Fang R, et al. (2020) Cutaneous manifestations of the Coronavirus Disease 2019 (COVID-19): A brief review. Dermatol Ther 2020: e13528.

9. Cohen JI (2013) Clinical practice: Herpes zoster. N Engl J Med 369(3): 255-263.

10. Tian S, Hu N, Lou J, Chen K, Kang X, et al. (2020) Characteristics of COVID-19 infection in Beijing. $\mathrm{J}$ Infect. 80(4): 401-406.

11. Heikkinen T, Järvinen A (2003) The common cold. Lancet 361(9351): 51-59.

12. Jin Y-H, Cai L, Cheng Z-S, Cheng H, Deng T, et al. (2020) A rapid advice guideline for the diagnosis and treatment of 2019 novel coronavirus (2019$\mathrm{nCoV}$ ) infected pneumonia (standard version). Mil Med Res 7(1): 4. 
13. Jia HP, Look DC, Shi L, Hickey M, Pewe L, et al. (2005) ACE2 receptor expression and severe acute respiratory syndrome coronavirus infection depend on differentiation of human airway epithelia. J Virol. 79(23): 14614-14621.

14. Vaira LA, Salzano G, De Riu G (2020) The importance of olfactory and gustatory disorders as early symptoms of coronavirus disease (COVID-19) Br J Oral Maxillofac Surg 58(5): 615-616.

15. Estébanez A, Pérez-Santiago L, Silva E, GuillenCliment S, García-Vázquez A, et al. (2020) Cutaneous manifestations in COVID-19: a new contribution. J Eur Acad Dermatol Venereol. 34(6): e250-e251.

16. Schwartz RA, Janniger CK (2020) Generalized pustular figurate erythema: A newly delineated severe cutaneous drug reaction linked with hydroxychloroquine. Dermatol Ther 33(3): e13380.
17. Recalcati S (2020) Cutaneous manifestations in COVID-19: a first perspective. J Eur Acad Dermatol Venereol. 34(5): e212-e213.c

18. Sachdeva M, Gianotti R, Shah M, Bradanini L, Tosi D, et al. (2020) Cutaneous manifestations of COVID-19: Report of three cases and a review of literature. J Dermatol Sci. 98(2): 75-81.

19. Demirbaş A, Elmas ÖF, Atasoy M, Türsen Ü, Lotti $\mathrm{T}$ (2020) A case of erythema multiforme major in a patient with COVID 19: The role of corticosteroid treatment. Dermatol ther. 33(6): e13899.

20. Santos JAD, Normando AGC, Carvalho da Silva RL, De Paula RM, Cembranel AC, et al. (2020) Oral mucosal lesions in a COVID-19 patient: New signs or secondary manifestations? Int J Infect Dis 97:326-328. 